\title{
Ideal Bose-gas in nonadditive statistics
}

\author{
Andrij Rovenchak \\ Department for Theoretical Physics, Ivan Franko National University of Lviv \\ 12 Drahomanov Str., Lviv UA-79005, Ukraine \\ E-mail: andrij.rovenchak@gmail.com
}

Received January 25, 2018, published online August 28, 2018

\begin{abstract}
The paper analyzes an approach to the generalization of the conventional Bose-Einstein statistics based on the nonadditive entropy of Tsallis. A detailed derivation of thermodynamic functions is presented. The calculations are made for the specific heat of two model systems, namely, the ideal three-dimensional gas obeying the nonadditive modification of the Bose-Einstein statistics and the system with linear excitation spectrum attempted as a qualitative approximation of liquid ${ }^{4}$ He thermodynamics.
\end{abstract}

Keywords: Bose-Einstein statistics, Tsallis entropy, nonadditive statistics, ideal Bose-gas, ${ }^{4} \mathrm{He}$.

\section{Introduction}

A generalization of the classical Boltzmann-Gibbs entropy was suggested by Tsallis [1]. The proposed nonadditive entropy might be relevant in descriptions of systems with long-range interactions, for non-Markovian processes or systems with "memory" and some others [2]. It has also been used in the description of complex systems beyond physics [3], for instance, to model DNA sequences [4], financial data [5] or distributions in linguistics [6].

In the present paper we will focus on the application of a nonadditive entropy for generalizations of quantum distributions [7-10]. For integrity, a presentation of the calculation scheme involving the density of states for a power-law excitation spectrum is followed by an introductory information about the Tsallis statistics and the description of one possible generalization of the Bose-distribution. A detailed mathematical derivation of thermodynamic quantities is complemented by calculations for two systems a three-dimensional ideal Bose-gas and a rough model of ${ }^{4} \mathrm{He}$.

We will consider the following simple scheme for the calculation of thermodynamic functions. The total number of particles is given as the sum of mean occupation numbers $n\left(\varepsilon_{j}, T, z\right)$ over all the states with energies $\varepsilon_{j}$,

$$
N=\sum_{j} n\left(\varepsilon_{j}, T, z\right)
$$

where $T$ is temperature and $z$ is fugacity related to the chemical potential $\mu$ via $z=\mathrm{e}^{\mu / T}$.

The dependence $z(T, N)$ being the solution of Eq. (1) can be used to calculate the total energy of the system

$$
E=\sum_{j} \varepsilon_{j} n\left(\varepsilon_{j}, T, z\right)
$$

as a function of temperature $T$ and the number of particles $N$. Further on, we can obtain the equation of state, calculate heat capacities and other thermodynamic quantities.

\section{Density of states}

For generality, let us consider a system of spinless bosons with the following elementary excitation spectrum

$$
\varepsilon_{p}=a p^{b}
$$

where $p \equiv|\mathbf{p}|$ is the momentum absolute value and $a, b$ are the spectrum parameters. In a $D$-dimensional space, $p=\sqrt{p_{1}^{2}+\ldots+p_{D}^{2}}$.

Suppose the particles reside in volume $\mathcal{V}_{D}$. If it is macroscopically large, the summation over states can be substituted by the integration over the phase space:

$$
N=\sum_{j} n\left(\varepsilon_{j}, T, z\right)=\int d \tau n\left(\varepsilon_{p}, T, z\right)
$$

where the phase space element

$$
d \tau=\frac{(d p)(d q)}{(2 \pi \hbar)^{D}}, \text { with }(d p)=d p_{1} \ldots d p_{D}, \quad(d q)=d q_{1} \ldots d q_{D}
$$

For particles with spin $\sigma$, additional multiplicity factor of $(2 \sigma+1)$ would occur.

This relation can be rewritten by introducing the density of states function $g(\varepsilon)$ :

$$
N=\int_{0}^{\infty} d \varepsilon g(\varepsilon) n(\varepsilon, T, z)
$$


There is no dependence on coordinates in the spectrum $\varepsilon_{p}$, so the integration over coordinates $(d q)$ is trivial:

$$
\int d \tau \ldots=\frac{1}{(2 \pi \hbar)^{D}} \int(d p) \int(d q) \ldots=\frac{\mathcal{V}_{D}}{(2 \pi \hbar)^{D}} \int(d p) \ldots
$$

Moreover, as the spectrum $\varepsilon_{p}$ depends only on the absolute value of the momentum $p$, the integration over momenta in (4) can be done in (hyper-)spherical coordinates, $d p_{1} \ldots d p_{D}=p^{D-1} d p d \Omega_{D}$ :

$$
N=\mathcal{V}_{D} \Omega_{D} \int_{0}^{\infty} d p p^{D-1} n\left(\varepsilon_{p}, T, z\right)
$$

where $\Omega_{D}=2 \pi^{D / 2} / \Gamma(D / 2)$ is the solid hyper-angle in the $D$-dimensional space.

Making the change of variables $\varepsilon=a p^{b}$ we finally obtain the density of states in the following form [11, p. 150]:

$$
g(\varepsilon)=\mathcal{V}_{D} \frac{2 \pi^{D / 2}}{\Gamma(D / 2)} \frac{a^{-s}}{b(2 \pi \hbar)^{D}} \varepsilon^{s-1}=\frac{\mathcal{V}_{D}}{\Gamma(D / 2+1)} \frac{s a^{-s}}{(2 \pi \sqrt{\hbar})^{D}} \varepsilon^{s-1},
$$

where $s=D / b$. In particular, for a gas of free particles with $\varepsilon_{p}=p^{2} / 2 m$ one gets [12]

$$
g(\varepsilon)=\frac{\mathcal{V}_{D}}{\Gamma(D / 2)}\left(\frac{m}{2 \pi \hbar^{2}}\right)^{D / 2} \varepsilon^{D / 2-1}
$$

Note that for a system of particles trapped to a harmonic potential $V\left(q_{1}, \ldots, q_{D}\right)=m\left(\omega_{1}^{2}+\ldots+\omega_{D}^{2}\right) / 2$ the density of states can be shown [12] to have the form

$$
g(\varepsilon)=\frac{1}{\Gamma(D)} \frac{1}{(\hbar \bar{\omega})^{D}} \varepsilon^{D-1}, \text { where } \bar{\omega}^{D}=\omega_{1} \ldots \omega_{D} .
$$

For convenience, we will introduce a shorthand notation for the constant in the density of states,

$$
\frac{1}{\Gamma(D / 2+1)} \frac{s a^{-s}}{(2 \pi \sqrt{\hbar})^{D}}=A \text {, so that } g(\varepsilon)=\mathcal{V}_{D} A \varepsilon^{s-1} \text {. }
$$

Equation (5) thus becomes as follows:

$$
\frac{N}{\mathcal{V}_{D}}=A \int_{0}^{\infty} d \varepsilon \varepsilon^{s-1} n(\varepsilon, T, z)
$$

Note that the consideration of thermodynamic function implies the so called thermodynamic limit, $N / \mathcal{V}_{D}=$ const as $N \rightarrow \infty$ and $\mathcal{V}_{D} \rightarrow \infty$. For a system of harmonic oscillators this condition is written as $N \bar{\omega}^{D}=$ const as $N \rightarrow \infty$ and $\bar{\omega} \rightarrow 0$.

\section{Nonadditive statistics of Tsallis}

In this section, we will briefly introduce an approach to generalize the conventional Boltzmann-Gibbs statistics.

For $W$ microstates with probabilities $p_{j}$ the generalized entropy as proposed by Constantino Tsallis [1] is given by
$S_{q}=\frac{1}{q-1}\left(1-\sum_{j=1}^{W} p_{j}^{q}\right)$, whereas $\sum_{j=1}^{W} p_{j}=1, q \in \mathbb{R}$.

In the limit of $q \rightarrow 1$ one easily recovers the BoltzmannGibbs entropy:

$$
p_{j}^{q-1}=\mathrm{e}^{(q-1) \ln p_{j}} \simeq 1+(q-1) \ln p_{j}
$$

so that

$$
S_{q}=\frac{1}{q-1}\left(1-\sum_{j=1}^{W} p_{j}^{q}\right)=\ldots=-\sum_{j=1}^{W} p_{j} \ln p_{j}
$$

as expected.

While the conventional entropy is additive, $S(A+B)=S(A)+S(B)$ in the case of a system split into two subsystems $A$ and $B$, the entropy from Eq. (12) can be shown to satisfy [2]

$$
S_{q}(A+B)=S_{q}(A)+S_{q}(B)+(1-q) S_{q}(A) S_{q}(B),
$$

meaning it is not an additive quantity. So, the parameter $q$ might be considered as a measure of nonadditivity.

Similarly to the conventional entropy, $S_{q}$ is maximal if all the probabilities are equal $\left(p_{j}=1 / W\right.$ for all $\left.j\right)$ :

$$
S_{q}=\frac{W^{1-q}-1}{1-q} \text {. }
$$

In the limit of $q \rightarrow 1$, the well-known relation $S=\ln W$ follows.

Using the so-called $q$-logarithm,

$$
\ln _{q} x \equiv \frac{x^{1-q}-1}{1-q}, \quad \text { whereas } \ln _{1} x=\ln x,
$$

one can write the Tsallis entropy in the following Boltzmann-like form:

$$
S_{q}=\ln _{q} W
$$

An inverse to the $q$-logarithm is given by the following function:

$$
\mathrm{e}_{q}^{x}= \begin{cases}\exp (x), & \text { for } q=1, \\ {[1+(1-q) x]^{1 /(1-q)},} & \text { for } q \neq 1 \text { and } 1+(1-q) x>0, \\ 0^{1 /(1-q)}, & \text { for } q \neq 1 \text { and } 1+(1-q) x \leq 0,\end{cases}
$$

which is known as the Tsallis q-exponential [13]. Its graphs are shown in Fig. 1.

There is a number of approaches based on the nonadditive entropy of Tsallis [14-17]. It appears in particular that the link between the entropy, energy, and temperature cannot be trivially replicated from the Boltzmann-Gibbs statistics. Strategies to generalize the Bose-Einstein and Fermi-Dirac distributions also vary [7,9,18,19]. 


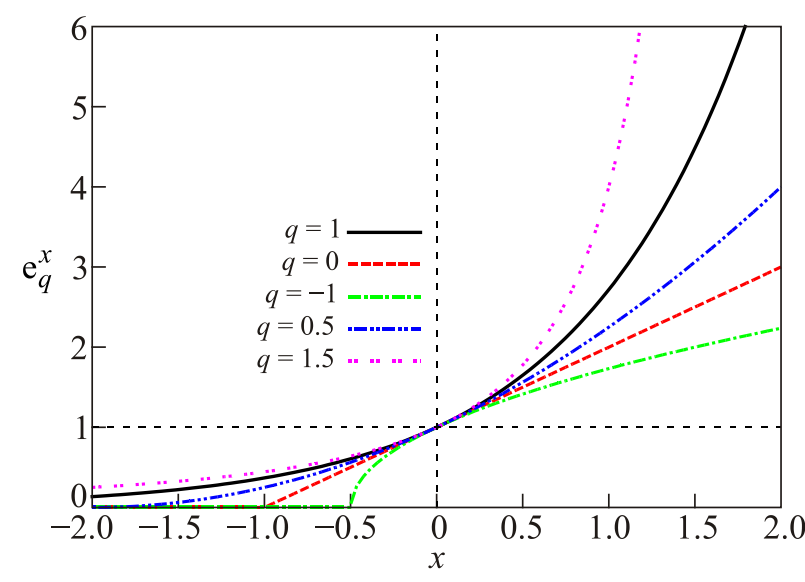

Fig. 1. (Color online) Tsallis $q$-exponential $\mathrm{e}_{q}^{x}$ for different values of the parameter $q$.

\section{Generalization of the Bose-statistics}

We will demonstrate a rather simple approach to generalize the quantum Bose-distribution. The Gibbs factor $\mathrm{e}^{(\varepsilon-\mu) / T}=z^{-1} \mathrm{e}^{\varepsilon / T}$ will be substituted to $z^{-1} \mathrm{e}_{q}^{\varepsilon / T}$. Note that the Tsallis $q$-exponentials do not obey the factorization rule,

$$
\mathrm{e}_{q}^{x+y} \neq \mathrm{e}_{q}^{x} \mathrm{e}_{q}^{y}
$$

but satisfy the following relations instead [20]:

$$
\left(\mathrm{e}_{q}^{x} \mathrm{e}_{q}^{y}\right)^{1-q}=\left(\mathrm{e}_{q}^{x+y}\right)^{1-q}+(1-q)^{2} x y,\left[\mathrm{e}_{q}^{x}\right]^{p}=\mathrm{e}_{1-(1-q) / p}^{p x} .
$$

We thus cannot relate the chemical potential and fugacity by a simple link, $z \neq \mathrm{e}_{q}^{\mu / T}$. On the other hand, such an approach facilitates comparisons between various types of fractional statistics via virial and cluster expansions [11], as shown for several nonadditive modifications in [21-23].

The generalized nonadditive Bose-distribution applied in this work is as follows

$$
n(\varepsilon, z, T)=\frac{1}{z^{-1} \mathrm{e}_{q}^{\varepsilon / T}-1} .
$$

There is only a seemingly subtle difference between this definition and, for instance, the one proposed in [18,24], where the fugacity was introduced as $z_{q}=\mathrm{e}_{q}^{\mu / T}$ and the occupation numbers were

$$
\tilde{n}(\varepsilon, \mu, T)=\frac{1}{\mathrm{e}_{q}^{(\varepsilon-\mu) / T}-1} .
$$

However, this difference significantly affects the high-temperature behavior, as we will see further.

\section{Critical temperature}

As $\mathrm{e}_{q}^{0}=1$, we observe a situation similar to that in ordinary Bose-distribution. Namely, the ground-state contribution is

$$
n(0, z, T)=\frac{1}{z^{-1}-1},
$$

which tends to infinity at $z \rightarrow 1$. Physically it means that the occupation of the ground state becomes macroscopically large, as in the ordinary Bose-Einstein condensation. On the other hand, this contribution is neglected if we consider the density of states (7) at $s>1$ since $g(\varepsilon)$ vanishes as $\varepsilon \rightarrow 0$. As a consequence, we should write the ground state occupation $N_{0} \equiv n(0, z, T)$ explicitly,

$$
N=N_{0}+A \mathcal{V}_{D} \int_{0}^{\infty} \frac{\varepsilon^{s-1} d \varepsilon}{z^{-1} \mathrm{e}_{q}^{\varepsilon / T}-1}
$$

where $N_{0}=0$ (i.e., it is a microscopic number, $N_{0} \ll N$ ) and $z<1$ for temperatures above some critical value $T_{C}$ and $N_{0}>0$ and $z=1$ below $T_{C}$. The critical temperature is defined by the condition

$$
N=A \mathcal{V}_{D} \int_{0}^{\infty} \frac{\varepsilon^{s-1} d \varepsilon}{\mathrm{e}_{q}^{\varepsilon / T_{C}}-1}
$$

Eliminating the temperature dependence in the integrand we obtain

$$
\frac{N}{\mathcal{V}_{D}}=A T_{c}^{S} \int_{0}^{\infty} \frac{x^{s-1} d x}{\mathrm{e}_{q}^{x}-1} .
$$

Evaluation of the integral in the definition of the critical temperature can be made similarly to the ordinary Bosestatistics. So,

$$
\int_{0}^{\infty} \frac{x^{s-1} d x}{\mathrm{e}_{q}^{x}-1}=\int_{0}^{\infty} d x x^{s-1} \frac{\left(\mathrm{e}_{q}^{x}\right)^{-1}}{1-\left(\mathrm{e}_{q}^{x}\right)^{-1}}=\int_{0}^{\infty} d x x^{s-1} \sum_{k=1}^{\infty}\left(\mathrm{e}_{q}^{x}\right)^{-k} .
$$

For $q=1$ this yields

$$
\int_{0}^{\infty} \frac{x^{s-1} d x}{\mathrm{e}^{x}-1}=\zeta(s) \Gamma(s)
$$

where Riemann's zeta-function reads (for $s>1$ ):

$$
\zeta(s)=\sum_{k=1}^{\infty} \frac{1}{k^{s}} .
$$

Other cases, namely $q<1$ and $q>1$, should be treated a bit differently.

For $q<1$, the argument $1+(1-q) x$ remains positive for all $x \geq 0$, so

$$
\begin{gathered}
\int_{0}^{\infty} \frac{x^{s-1} d x}{\mathrm{e}_{q}^{X}-1}=\sum_{k=1}^{\infty} \int_{0}^{\infty} d x x^{s-1}[1+(1-q) x]^{-\frac{k}{1-q}}= \\
=\frac{1}{(1-q)^{s}} \sum_{k=1}^{\infty} \mathrm{B}\left(s, \frac{k}{1-q}-s\right),
\end{gathered}
$$

where the beta-function $\mathrm{B}(u, v)=\Gamma(u) \Gamma(v) / \Gamma(u+v)$. 
For $q>1$, one has to set the upper limit of integration $x_{0}=1 /(q-1)$, where $1+(1-q) x$ becomes negative. So,

$$
\begin{aligned}
\int_{0}^{\infty} \frac{x^{s-1} d x}{\mathrm{e}_{q}^{X}-1} & =\sum_{k=1}^{\infty} \int_{0}^{x_{0}} d x x^{s-1}[1+(1-q) x]^{-\frac{k}{1-q}}= \\
= & \frac{1}{(q-1)^{s}} \sum_{k=1}^{\infty} \mathrm{B}\left(s, \frac{k}{q-1}+1\right)
\end{aligned}
$$

To be consistent with the $q=1$ case we can formally introduce a $q$-generalized zeta-function in the following manner, cf. [18,24]:

$$
\zeta_{q}(s)= \begin{cases}\zeta(s) & \text { for } q=1, \\ \frac{1}{(1-q)^{s} \Gamma(s)} \sum_{k=1}^{\infty} \mathrm{B}\left(s, \frac{k}{1-q}-s\right) & \text { for } q<1, \\ \frac{1}{(q-1)^{s} \Gamma(s)} \sum_{k=1}^{\infty} \mathrm{B}\left(s, \frac{k}{q-1}+1\right) & \text { for } q>1,\end{cases}
$$

so that

$$
\int_{0}^{\infty} \frac{x^{s-1} d x}{\mathrm{e}_{q}^{X}-1}=\zeta_{q}(s) \Gamma(s)
$$

with the definition of the critical temperature in Eq. (25) becoming consistent with the ordinary Bose-condensation temperature:

$$
\frac{N}{\mathcal{V}_{D}}=A T_{c}^{s} \zeta_{q}(s) \Gamma(s)
$$

As a bonus from such a form, the factor of $\Gamma(s)$ explicitly present in the above formula cancels the same factor in the denominators of the density of states given by Eqs. (8), (9).

Note that from the asymptotic behavior of the beta-function at large $v$ while keeping $u$ fixed,

$$
\mathrm{B}(u, v) \sim \Gamma(u) v^{-u},
$$

one easily recovers Eq. (26) from $\zeta_{q}(s)$ in the limits of $q \rightarrow 1$. Unfortunately, a general closed-form expression for $\zeta_{q}(s)$ without infinite summations has not been found.

\section{Low temperature}

For temperatures below the critical one $T_{c}$, where $z=1$, the calculations are quite simple. From Eq. (23) using (30) we obtain

$$
N=N_{0}+A \mathcal{V}_{D} \int_{0}^{\infty} \frac{\varepsilon^{s-1} d \varepsilon}{\mathrm{e}_{q}^{\varepsilon / T}-1}=N_{0}+A \mathcal{V}_{D} T^{s} \zeta_{q}(s) \Gamma(s),
$$

or applying the critical temperature definition (31),

$$
\frac{N_{0}}{N}=1-\left(\frac{T}{T_{C}}\right)^{S}
$$

which coincides with the expression for the condensate fraction (relative number of particles with zero momenta) in ordinary ideal Bose-gas.

The calculation of energy is also simple

$$
E=A \mathcal{V}_{D} \int_{0}^{\infty} \frac{\varepsilon^{s} d \varepsilon}{\mathrm{e}_{q}^{\varepsilon / T}-1}=A \mathcal{V}_{D} T^{s+1} \zeta_{q}(s+1) \Gamma(s+1),
$$

which can be written as

$$
E=s \frac{\zeta_{q}(s+1)}{\zeta_{q}(s)} N T\left(\frac{T}{T_{c}}\right)^{s},
$$

again being consistent with the energy of an ideal Bose-gas at $q=1$.

The isochoric heat capacity is thus proportional to $T^{S}$ :

$$
C_{V}=\left(\frac{\partial E}{\partial T}\right)_{V}=s(s+1) \frac{\zeta_{q}(s+1)}{\zeta_{q}(s)} N\left(\frac{T}{T_{C}}\right)^{s} .
$$

The calculations above $T_{c}$ would require a more elaborated approach involving in particular a $q$-generalization of the polylogarithm function $\mathrm{Li}_{q, s}(z)$. For instance, at $q<1$, the number of particles is defined by the integral

$$
\begin{gathered}
\int_{0}^{\infty} \frac{x^{s-1} d x}{z^{-1} \mathrm{e}_{q}^{X}-1}=\sum_{k=1}^{\infty} z^{k} \int_{0}^{\infty} d x x^{s-1}[1+(1-q) x]^{-\frac{k}{1-q}}= \\
=\frac{1}{(1-q)^{s}} \sum_{k=1}^{\infty} z^{k} \mathrm{~B}\left(s, \frac{k}{1-q}-s\right) \equiv \Gamma(s) \mathrm{Li}_{q, s}(z)
\end{gathered}
$$

and similarly for $q>1$. This new function reduces to the ordinary polylogarithm at $q \rightarrow 1$,

$$
\operatorname{Li}_{1, s}(z) \equiv \operatorname{Li}_{s}(z)=\sum_{k=1}^{\infty} \frac{z^{k}}{k^{s}} .
$$

However, no simple recursive relations can be written for derivatives $\mathrm{Li}_{q, s}(z)$, unlike the undeformed case $(q=1)$ or the approach of $[18,24]$. Consequently, only a cumbersome expression would be obtained for the heat capacity calling rather for simple numerical evaluation instead.

In particular, one can show that the behavior of the isochoric heat capacity at the critical temperature is qualitatively the same as in the ordinary ideal Bose-system [25]. Namely, $C_{V}$ is continuous for $1<s<3 / 2$, there is a cusp on the $C_{V}$ curve for $s=3 / 2$, and there is a discontinuity (a finite jump) of $C_{V}$ for $s>3 / 2$.

\section{High temperatures and classical limit}

At high temperatures, fugacity tends to zero, so approximately

$$
N=z \mathcal{V}_{D} A \int_{0}^{\infty} d \varepsilon \varepsilon^{s-1}\left(\mathrm{e}_{q}^{\varepsilon / T}\right)^{-1}
$$


In the same approximation, energy is given by

$$
E=z \mathcal{V}_{D} A \int_{0}^{\infty} d \varepsilon \varepsilon^{s}\left(\mathrm{e}_{q}^{\varepsilon / T}\right)^{-1}
$$

The change of variables $x=\varepsilon / T$ yields

$$
N=z T^{S} \mathcal{V}_{D} A \int_{0}^{\infty} d x x^{s-1}\left(\mathrm{e}_{q}^{x}\right)^{-1}
$$

and

$$
E=z T^{s+1} \mathcal{V}_{D} A \int_{0}^{\infty} d x x^{s}\left(\mathrm{e}_{q}^{x}\right)^{-1}
$$

The integration in the above expressions is made using the same approach as applied in Eqs. (27) and (28), considering the cases of $q<1$ and $q>1$ separately and keeping only first terms in the sum.

So, for $q<1$

$$
N=\frac{z T^{s} \mathcal{V}_{D} A}{(1-q)^{s}} \mathrm{~B}\left(s, \frac{1}{1-q}-s\right)=z T^{s} \mathcal{V}_{D} A \frac{\Gamma(s) \Gamma\left(\frac{1}{1-q}-s\right)}{(1-q)^{s} \Gamma\left(\frac{1}{1-q}\right)}
$$

and

$$
\begin{aligned}
& E=\frac{z T^{s+1} \mathcal{V}_{D} A}{(1-q)^{s+1}} \mathrm{~B}\left(s+1, \frac{q}{1-q}-s\right)= \\
& =z T^{s+1} \mathcal{V}_{D} A \frac{\Gamma(s+1) \Gamma\left(\frac{q}{1-q}-s\right)}{(1-q)^{s+1} \Gamma\left(\frac{1}{1-q}\right)} .
\end{aligned}
$$

Eliminating fugacity between the above two equations we obtain

$$
E=\frac{s}{q(s+1)-s} N T
$$

For $q>1$ we have

$$
N=\frac{z T^{s} \mathcal{V}_{D} A}{(q-1)^{s}} \mathrm{~B}\left(s, \frac{q}{q-1}\right)=z T^{s} \mathcal{V}_{D} A \frac{\Gamma(s) \Gamma\left(\frac{q}{q-1}\right)}{(q-1)^{s} \Gamma\left(\frac{q}{q-1}+s\right)}
$$

and

$$
\begin{gathered}
E=\frac{z T^{s+1} \mathcal{V}_{D} A}{(q-1)^{s+1}} \mathrm{~B}\left(s+1, \frac{q}{q-1}\right)= \\
=z T^{s+1} \mathcal{V}_{D} A \frac{\Gamma(s+1) \Gamma\left(\frac{q}{q-1}\right)}{(q-1)^{s+1} \Gamma\left(\frac{q}{q-1}+s+1\right)} .
\end{gathered}
$$

These again yields for energy the same result as for $q<1$, namely:

$$
E=\frac{s}{q(s+1)-s} N T
$$

In the case of $q=1$ it reduces to the well-known classical limit for energy in the form $E=s N T$. For instance, a gas of free particles $(b=2)$ in three dimensions $(D=3$ and $s=D / b=3 / 2$ ) yields the textbook expression:

$$
E=\frac{3}{2} N T
$$

The isochoric heat capacity at hight temperatures is thus

$$
C_{V}=\left(\frac{\partial E}{\partial T}\right)_{V}=\frac{s}{q(s+1)-s} N .
$$

Note that the nonadditivity parameter must satisfy the condition $q>s /(s+1)$ ensuring that this classical limit remains positive.

\section{Results in three dimensions}

To demonstrate the application of the developed approach we have performed calculations for two systems. The first one is the three-dimensional gas of free particles while the second one might be considered as a rough model for liquid ${ }^{4} \mathrm{He}$.

The density of states (8) of the free 3D system is given by

$$
g(\varepsilon)=\frac{V}{\Gamma(3 / 2)}\left(\frac{m}{2 \pi \hbar^{2}}\right)^{3 / 2} \varepsilon^{3 / 2-1}
$$

with $V$ standing for the 3D volume $V \equiv \mathcal{V}_{3}$. The critical temperature is defined by

$$
\frac{N}{V}=\left(\frac{m T_{C}}{2 \pi \hbar^{2}}\right)^{3 / 2} \zeta_{q}\left(\frac{3}{2}\right) .
$$

The plots for the specific heat $C_{V} / N$ are shown in Fig. 2 for several values of the nonadditivity parameter $q$.

When comparing the obtained dependences with [18,24] we can see the discrepancies in the high-temperature behavior of the specific heat, especially for $q<1$, where the approach of $[18,24]$ yielded a minimum on the $C_{V}$ curve at $T>T_{C}$. Moreover, it can be shown that the asymptotic is $C_{V} \propto T^{(1-q) s /[1-(1-q) s]}$ (see Appendix), not the expected classical $C_{V} \rightarrow$ const. These differences demonstrate the importance of the fugacity definition in the deformed statistics.

The second system was attempted as a rough model for the specific heat of ${ }^{4} \mathrm{He}$ [26-28], which was historically the first Bose-system extensively studied both experimentally and theoretically.

The phonon branch of the elementary excitation spectrum in ${ }^{4} \mathrm{He}$ leads to the temperature dependence of the iso- 


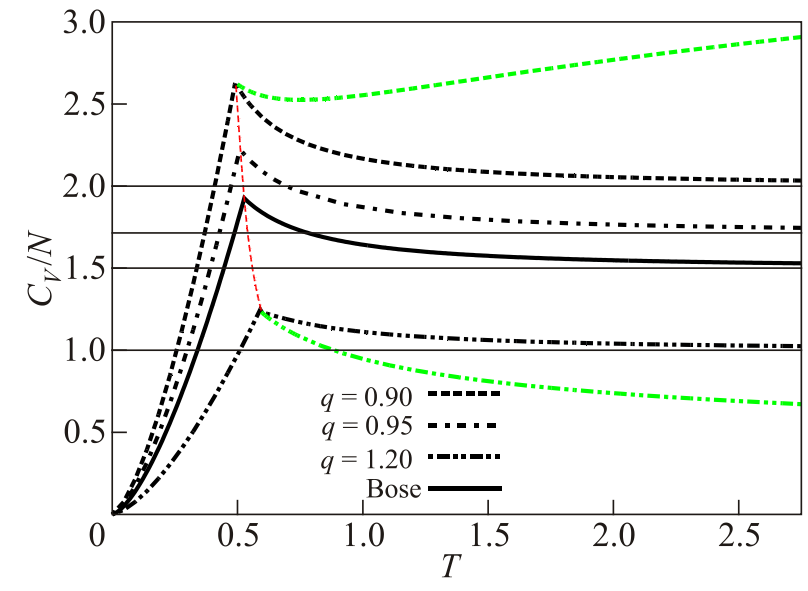

Fig. 2. (Color online) Specific heat of the three-dimensional ideal Bose-gas with nonadditive statistics compared to the ordinary Bose-gas. Green lines show the results consistent with the approach of $[18,24]$. Red dashed line joins the cusps of the respective curves corresponding to critical temperatures. For convenience, the units of temperature and energy are fixed by $\frac{N}{V}\left(\frac{2 \pi \hbar^{2}}{m}\right)^{3 / 2}=1$.

choric heat capacity at low temperatures as $T^{3}$, like in the Debye model of the solid. So, it fixes the value of $s=3$. The classical limit for the heat capacity of a monoatomic gas, which is the case of helium, equals $C_{V}=3 N / 2$. Thus, from Eq. (48) we obtain

$$
C_{V}=\frac{s}{q(s+1)-s} N=\frac{3}{2} N
$$

yielding $q=5 / 4$.

Generally, one can expect that in realistic physical systems the value of $q$ does not significantly deviate from unity. For instance, an effective accounting for interactions in a weakly-interacting Bose system can be achieved with $q \simeq 0.978$ [21]. Larger deviations, as obtained from (52), can occur if strong interactions come into play.

The results of calculations are shown in Fig. 3. Note the discontinuity at the critical temperature occurring both in real ${ }^{4} \mathrm{He}$ and in the model nonadditive system, but not in the ordinary 3D ideal Bose-gas.

As one can see, the specific heat curves are plotted in the relative temperature scale. The reason is that the critical temperature of the ideal Bose gas with ${ }^{4} \mathrm{He}$ mass and density is $T_{C}=3.14 \mathrm{~K}$ versus the lambda-transition temperature in liquid ${ }^{4} \mathrm{He} T_{\lambda}=2.17 \mathrm{~K}$. Such a discrepancy can be solved in particular by introducing the notion of an effective mass [30] calculated within various approaches [31-35].

It appears that within the proposed nonadditive model we cannot achieve simultaneous agreement between the critical temperature and the speed of sound determining the factor at $T^{3}$ in the specific heat as both these values are defined by a single parameter $A$ in the density of states.

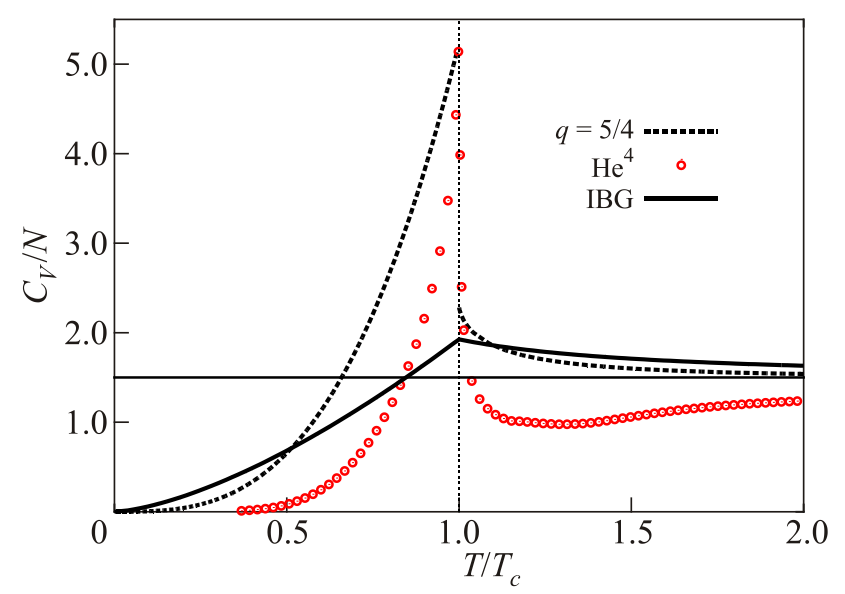

Fig. 3. (Color online) Specific heat of ${ }^{4} \mathrm{He}$ (circles, data from [29]) compared to the ordinary 3D ideal Bose-gas (IBG) and the nonadditive model with $q=5 / 4, s=3$. The data are plotted in the temperature scale relative to the critical temperatures for each system.

The values of the $q$-generalized zeta function appearing in calculations are as follows:

$$
\begin{gathered}
\zeta_{5 / 4}(3)=\frac{16}{3}(\ln 8-2)=0.423688 \ldots, \\
\zeta_{5 / 4}(4)=\frac{32}{3}(\ln 64-\pi-1)=0.184431 \ldots,
\end{gathered}
$$

These yield upon putting $T_{C}=2.17 \mathrm{~K}$ in (31) and (36):

$$
\frac{1}{A} \frac{N}{V} \simeq 65\left[\mathrm{~K}^{3}\right]
$$

and

$$
\frac{C_{V}}{N}=s(s+1) \frac{\zeta_{q}(s+1)}{\zeta_{q}(s)}\left(\frac{T}{T_{c}}\right)^{s} \simeq 0.51 T^{3}
$$

with temperature in Kelvins. For the specific heat of a bosonic phonon system with spectrum $\varepsilon_{p}=c p$ one obtains:

$$
\frac{C_{V}}{N}=\frac{16}{15} \pi^{5} \frac{V}{N} \frac{T^{3}}{(h c)^{3}},
$$

where $c$ is the speed of sound and $h \equiv 2 \pi \hbar$. With ${ }^{4} \mathrm{He}$ parameters, $N / V=0.02185 \AA^{-3}$ and $c=237 \mathrm{~m} / \mathrm{s}$ this gives

$$
\frac{C_{V}}{N} \simeq 0.01 T^{3} \text {. }
$$

The discrepancy between Eqs. (56) and (58) means that the speed of sound in the model system is about $64 \mathrm{~m} / \mathrm{s}$. Such a difference is explained by the fact that the $T^{3}$ behavior of the heat capacity holds only at very low temperatures $(T<0.6 \mathrm{~K})$ and for higher temperatures other types of excitations, in particular the so called rotons, should be considered as well [26,27]. In order to adjust the proposed 
nonadditive model for ${ }^{4}$ He one may also try to define the value of $s$ from the specific heat behavior at temperatures closer to the critical one, where $C_{V} \propto T^{6 \div 7}$ [27].

\section{Conclusions}

To summarize, we have proposed an approach to generalize the Bose-Einstein distribution using nonadditive statistics of Tsallis. Detailed derivations of thermodynamic functions have been given for a $D$-dimensional system with the elementary excitation spectrum $\varepsilon_{p}=a p^{b}$. A phenomenon being an analog of the Bose-condensation has been detected and the corresponding critical temperature has been calculated. The low- and high-temperature behavior of energy and specific heat has been analyzed.

Both analytical and numerical calculations have been made for two model systems. From the analysis of the first one, an ideal 3D gas, the importance of the fugacity definition has been revealed in comparison with other approaches. The second system could be a rough model for the specific heat of ${ }^{4} \mathrm{He}$. Its limitations have been briefly discussed. In particular, it became clear that a single nonadditivity parameter $q$ is not sufficient to agree both the value of the critical temperature and the speed of sound with experimental data.

The results of the present work would be useful in applications of unconventional types of statistics as effective models of real physical systems.

\section{Acknowledgment}

This work was partly supported by Project FF-30F (No. 0116U001539) from the Ministry of Education and Science of Ukraine.

\section{Appendix A}

Consider the expression for the number of particles $N$ and energy $E$ in the approach of $[18,24]$ :

$$
N=\int_{0}^{\infty} \frac{g(\varepsilon) d \varepsilon}{\mathrm{e}_{q}^{(\varepsilon-\mu) / T}-1}, \quad E=\int_{0}^{\infty} \frac{\varepsilon g(\varepsilon) d \varepsilon}{\mathrm{e}_{q}^{(\varepsilon-\mu) / T}-1}
$$

Note that these authors originally used a mirrored definition of the $q$-exponential with the parameter $\tilde{q}$ related to the parameter $q$ of the present paper via $\tilde{q}-1=1-q$.

Upon making a numerical analysis one can conclude that in the limit $T \rightarrow \infty$ the chemical potential has the following behavior:

$$
\frac{\mu}{T}=-\frac{|\mu|}{T} \rightarrow-\infty
$$

In such a limit, the unity in the denominator of the expression for occupation numbers can be neglected, so

$$
N \simeq \int_{0}^{\infty} d \varepsilon g(\varepsilon)\left[\mathrm{e}_{q}^{(\varepsilon-\mu) / T}\right]^{-1}=\mathcal{V}_{D} A T^{S} \int_{0}^{\infty} d x x^{S-1}\left[\mathrm{e}_{q}^{x+|\mu| / T}\right]^{-1}
$$

For definiteness, we will consider $q<1$. The number of particles is thus

$$
\begin{aligned}
& N \simeq \mathcal{V}_{D} A T^{S} \int_{0}^{\infty} d x x^{s-1}\left[1+(1-q)\left(x+\frac{|\mu|}{T}\right)\right]^{-\frac{1}{1-q}} \simeq \\
& \simeq \mathcal{V}_{D} A T^{s}\left((1-q) \frac{|\mu|}{T}\right)^{\frac{1}{q-1}} \int_{0}^{\infty} x^{s-1}\left(\frac{T}{|\mu|} x+1\right)^{-\frac{1}{1-q}}= \\
&=\mathcal{V}_{D} A|\mu|^{S}\left((1-q) \frac{|\mu|}{T}\right)^{\frac{1}{q-1}} \mathrm{~B}\left(s, \frac{1}{1-q}-s\right) .
\end{aligned}
$$

In the same fashion the energy is

$$
\begin{aligned}
& E \simeq \mathcal{V}_{D} A T^{s+1} \int_{0}^{\infty} d x x^{s}\left[1+(1-q)\left(x+\frac{|\mu|}{T}\right)\right]^{-\frac{1}{1-q}} \simeq \\
& \simeq \mathcal{V}_{D} A|\mu|^{s+1}\left((1-q) \frac{|\mu|}{T}\right)^{\frac{1}{q-1}} \mathrm{~B}\left(s+1, \frac{1}{1-q}-s-1\right) .
\end{aligned}
$$

So,

$$
\frac{E}{N}=\frac{\mathrm{B}\left(s+1, \frac{1}{1-q}-s-1\right)}{\mathrm{B}\left(s, \frac{1}{1-q}-s\right)}|\mu|,
$$

while

$$
|\mu|=\left[\frac{N}{\mathcal{V}_{D}} \frac{1}{A} \frac{(1-q)^{\frac{1}{1-q}}}{\mathrm{~B}\left(s, \frac{1}{1-q}-s\right)}\right]^{\frac{1}{1-s(1-q)}}
$$

Therefore, the specific heat depends on temperature as

$$
\frac{C_{V}}{N} \propto T^{\frac{(1-q) s}{1-s(1-q)}}=T^{\gamma}
$$

Such a relation can be shown to hold for $q>1$ as well. The numerical values are, in particular, for $s=3 / 2$ (see also Fig. 4):

$$
\begin{array}{ll}
q=0.9: & \gamma=+\frac{3}{17}, \quad C_{V} \propto T^{+0.176 \ldots,} \\
q=1.2: & \gamma=-\frac{3}{13}, \quad C_{V} \propto T^{-0.230 \ldots .}
\end{array}
$$




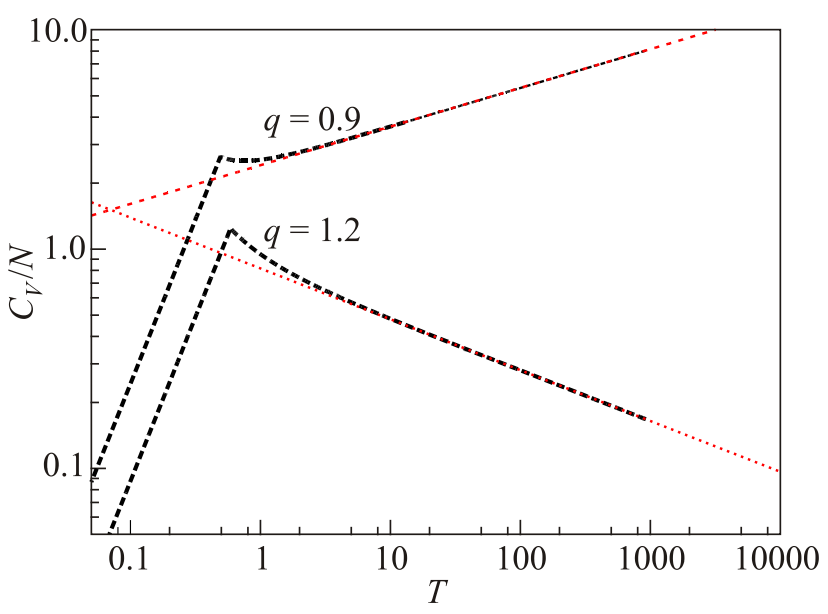

Fig. 4. (Color online) Specific heat of the three-dimensional ideal Bose-gas with nonadditive statistics according to the approach of $[18,24]$. Red dashed lines show the high-temperature behavior (A8). The units of temperature are fixed as in Fig. 2.

1. C. Tsallis, J. Stat. Phys. 52, 479 (1988).

2. Nonextensive Statistical Mechanics and Its Applications, S. Abe and Y. Okamoto (eds.), Springer, Berlin (2001).

3. Nonextensive Entropy: Interdisciplinary Applications, M. Gell-Mann and C. Tsallis (eds.), Oxford University Press, New York (2004).

4. G.P. Pavlos, L.P. Karakatsanis, A.C. Iliopoulos, E.G. Pavlos, M.N. Xenakis, P. Clark, J. Duke, and D.S. Monos, Physica A 438, 188 (2015).

5. G. Ruiz and A.F. de Marcos, Eur. Phys. J. B 91, 1 (2018).

6. A. Rovenchak and S. Buk, J. Quant. Ling. 25, 1 (2018).

7. F. Büyükklç, D. Demirhan, and A. Güleç, Phys. Lett. A 197, 209 (1995).

8. B. Tanatar, Phys. Rev. E 65, 046105 (2002).

9. H.H. Aragão-Rêgo, D.J. Soares, L.S. Lucena, L.R. da Silva, E.K. Lenzi, and K.S. Fa, Physica A 317, 199 (2003).

10. H. Mohammadzadeh, F. Adli, and S. Nouri, Phys. Rev. E 94, 062118 (2016) .

11. A. Khare, Fractional Statistics and Quantum Theory, World Scientific, Singapore (2005).

12. L. Salasnich, Intern. J. Mod. Phys. B 14, 405 (2000).

13. C. Tsallis, Química Nova 17, 468 (1994).

14. E.M.F. Curado and C. Tsallis, J. Phys. A: Math. Gen. 24, L69 (1991).

15. C. Tsallis, R.S. Mendes and A.R. Plastino, Physica A 261, 534 (1998).

16. C. Tsallis, Introduction to Nonextensive Statistical Mechanics: Approaching a Complex World, Springer, New York (2009).

17. G. Wilk and Z. Włodarczyk, Physica A 413, 53 (2014).

18. J. Chen, Z. Zhang, G. Su, L. Chen, and Y. Shu, Phys. Lett. A 300, 65 (2002).

19. K.-M. Shen, B.-W. Zhang and E.-K. Wang, Physica A 487, 215 (2017).

20. T. Yamano, Physica A 305, 486 (2002).

21. A. Rovenchak, Phys. Rev. A 89, 052116 (2014).

22. A. Rovenchak, Eur. Phys. J. B 87, 175 (2014).
23. M.Y. Hornetska and A.A. Rovenchak, Ukr. J. Phys. 61, 168 (2016).

24. C. Ou and J. Chen, Phys. Rev. E 68, 026123 (2003).

25. L. Chen, Z. Yan, M. Li, and C. Chen, J. Phys. A 31, 8289 (1998).

26. R.A. Hull, K.R. Wilkinson, and J. Wilks, Proc. Phys. Soc. Sec. A 64, 379 (1951).

27. H.C. Kramers, J.D. Wasscher, and C.J. Gorter, Physica 18, 329 (1952).

28. O.V. Lounasmaa, Cryogenics 1, 212 (1961).

29. V.D. Arp, R.D. McCarty, and D.G. Friend, Natl. Inst. Stand. Technol. Tech. Note 1334 (revised) (1998).

30. R.P. Feynman, Phys. Rev. 91, 1291 (1953).

31. A. Isihara and T. Samulski, Phys. Rev. B 16, 1969 (1977).

32. A.A. Rovenchak, Fiz. Nizk. Temp. 29, 145 (2003) [Low Temp. Phys. 29, 105 (2003)].

33. K.A. Gernoth, M. Serhan, and M.L. Ristig, Phys. Rev. B 78, 054513 (2008).

34. I.O. Vakarchuk, V.S. Pastukhov, and R.O. Prytula, Ukr. Phys. J. 57, 1214 (2012).

35. I.O. Vakarchuk, O.I. Hryhorchak, V.S. Pastukhov, and R.O. Prytula, Ukr. J. Phys. 61, 29 (2016).

\section{Ідеальний бозе-газ з неадитивною статистикою}

\section{А. Ровенчак}

Проаналізовано підхід до узагальнення традиційної статистики Бозе-Ейнштейна на основі неадитивної ентропії Цалліса. Подано докладне виведення термодинамічних функцій. Зроблено розрахунки для питомої теплоємності двох модельних систем, а саме: ідеального тривимірного газу, що підкоряється неаддитивній модифікації статистики Бозе-Ейнштейна, та системи 3 лінійним спектром елементарних збуджень, прийнятої за якісне наближення термодинаміки рідкого ${ }^{4} \mathrm{He}$.

Ключові слова: статистика Бозе-Ейнштейна, ентропія Цалліca, неадитивна статистика, ідеальний бозе-газ, ${ }^{4} \mathrm{He}$.

\section{Идеальный бозе-газ с неаддитивной статистикой}

\section{А. Ровенчак}

Проанализирован подход к обобщению традициионной статистики Бозе-Эйнштейна на основе неаддитивной энтропии Цаллиса. Представлен подробный вывод термодинамических функций. Сделаны расчеты для удельной теплоемкости двух модельных систем, а именно: идеального трехмерного газа, подчиняющегося неаддитивной модификации статистики Бозе-Эйнштейна, и системы с линейным спектром элементарных возбуждений, принятой как качественное приближение термодинамики жидкого ${ }^{4} \mathrm{He}$.

Ключевые слова: статистика Бозе-Эйнштейна, энтропия Цаллиса, неаддитивная статистика, идеальный бозе-газ, ${ }^{4} \mathrm{He}$. 